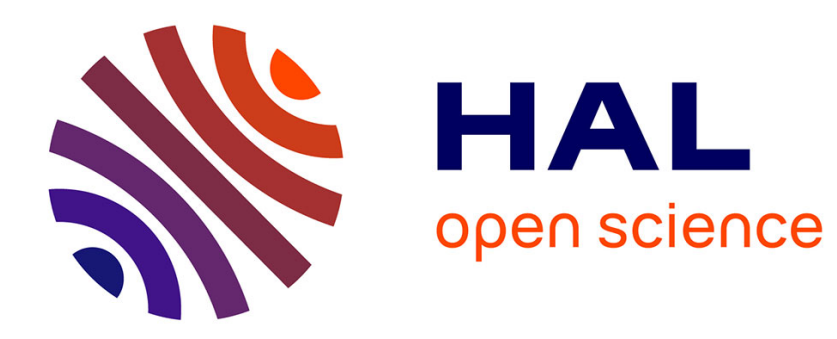

\title{
Projet d'un système de détente hydraulique pour une chambre à bulles à liquide lourd
}

\author{
A. Lefrançois
}

\section{To cite this version:}

A. Lefrançois. Projet d'un système de détente hydraulique pour une chambre à bulles à liquide lourd. Revue de Physique Appliquée, 1969, 4 (2), pp.239-240. 10.1051/rphysap:0196900402023900 . jpa00243240

\section{HAL Id: jpa-00243240 https://hal.science/jpa-00243240}

Submitted on 1 Jan 1969

HAL is a multi-disciplinary open access archive for the deposit and dissemination of scientific research documents, whether they are published or not. The documents may come from teaching and research institutions in France or abroad, or from public or private research centers.
L'archive ouverte pluridisciplinaire HAL, est destinée au dépôt et à la diffusion de documents scientifiques de niveau recherche, publiés ou non, émanant des établissements d'enseignement et de recherche français ou étrangers, des laboratoires publics ou privés. 


\title{
PROJET D'UN SYSTÈME DE DÉTENTE HYDRAULIQUE POUR UNE GHAMBRE A BULLES A LIQUIDE LOURD
}

\author{
A. LEFRANÇOIS, \\ C.E.R.N., Genève.
}

\begin{abstract}
Résumé. - La détente est provoquée par le mouvement d'un piston agissant sur le liquide de la chambre par l'intermédiaire d'un coussin d'huile et d'une membrane. Le piston est commandé pendant la détente par deux vannes indépendantes; il est maintenu ensuite en position "compression" par un servomécanisme de position.
\end{abstract}

Abstract. - The expansion is caused by the movement of a piston acting on the liquid of the chamber through an oil cushion and a membrane. The piston is operated during the expansion by means of two independent valves; it is then maintained in position of "compression" by means of a positioning servomechanism.

I. Introduction. - Notre chambre à bulles à liquide lourd de $1,1 \mathrm{~m}^{3}$ a été actionnée jusqu'à présent par un système de détente pneumatique tout à fait classique pour ce type de chambre et conçu pour un taux de répétition maximal de $1,8^{\prime \prime}$ environ. Le problème s'étant posé de disposer d'un système capable d'un taux de répétition de $1^{\prime \prime}$ analogue au nouveau taux de la machine, il a été décidé d'entreprendre la construction d'un système de détente hydraulique, plus souple et mieux adapté aux fréquents déplacements de notre chambre.

II. Mode de transfert de la puissance hydraulique au liquide de la chambre. - Dans la solution retenue,

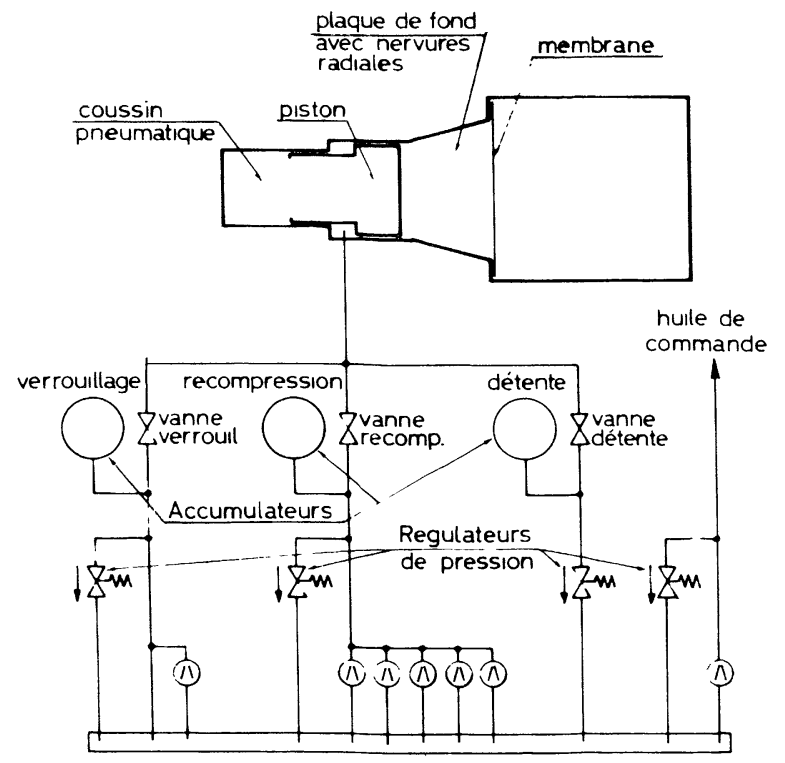

FIG. 1. - Schéma d'ensemble. l'huile agit sur un piston en contact avec le liquide de la chambre par l'intermédiaire d'un volume d'huile et d'une membrane. Les orifices de passage de l'huile à travers la plaque de fond de la chambre sont limités par la surface nécessaire pourleflux magnétique ( fig. 1).

III. Application du principe de l'oscillation naturelle au système de détente. - III.1. DIFFÉRENTES VARIANTES. - Nous avons adopté dès le départ un système commandé (système résonnant) comportant un piston à simple effet alimenté hydrauliquement sous deux niveaux de pression différents à travers deux vannes indépendantes. On peut distinguer deux variantes fondamentales selon l'orientation de la face du piston sur laquelle agit l'huile de commande : dans le sens de la détente pour le système à impulsion de détente et dans le sens de la recompression pour le système à verrouillage. Nous avons choisi la deuxième solution parce qu'elle se traduit, au point de vue cycle réel de pression sur la face de commande du piston (compte tenu des pertes), par un moins grand débit d'huile à pression élevée ( fig. 2) et qu'elle conduit ainsi à une moindre fatigue des pompes hydrauliques.

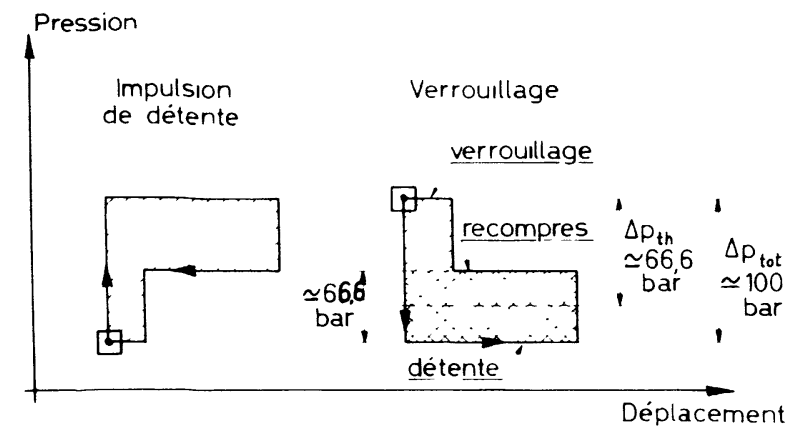

FiG. 2. - Cycles de pression. 
III . 2. Dimensionnement. - La différence de pression représentée par $\Delta p_{\text {th }}$ sur le diagramme des cycles de pression correspond à la moitié de la différence de pression $\Delta p_{\mathrm{L}}$ dans le liquide entre l'état comprimé et la détente, compte tenu d'un facteur d'amplification égal au rapport des surfaces du piston côté huile intermédiaire et côté vannes. Nous avons choisi un rapport de surfaces égal à 0,15 qui donne $\Delta p_{\text {th }}=66,6$ bars pour $\Delta p_{\mathrm{L}}=20$ bars, ce qui permettrait d'opérer avec un liquide à pression de vapeur plus élevée que le propane. La différence de pression maximale apparaissant sur le cycle $\left(\Delta p_{\text {tot }}\right)$ doit être choisie en fonction de $\Delta p_{\text {th }}$ de façon à ce qu'il soit fourni au piston une certaine énergie correspondant aux pertes (frottement du piston, pertes de charges dans l'huile, pertes par courants de Foucault, pertes dans la chambre). Pour une valeur de $\Delta p_{\text {tot }} \simeq 100$ bars et une variation de volume dans la chambre de 251 (volume d'huile déplacé derrière le piston $3,75 \mathrm{l}$ ), on obtient sur la partie quadrillée du diagramme un apport d'énergie de $25000 \mathrm{~J}$ par détente, qui doit être largement suffisant. Cette valeur de $\Delta p_{\text {tot }}$, soit 100 bars, permet de travailler à l'aise, compte tenu des pertes de charge, avec des pompes fonctionnant normalement à 140 bars.

IV. Réalisation. Système de commande hydraulique. - IV.1. Piston. - Le piston est en alliage d'aluminium. Son diamètre sera $0,620 \mathrm{~m}$ côté huile intermédiaire et $0,572 \mathrm{~m}$ côté coussin pneumatique, son poids environ $250 \mathrm{~kg}$. Des dash-pots hydrauliques dont l'un est réglable en position amortiront les mouvements en cas de fausse manœuvre. La course sera d'environ $8 \mathrm{~cm}$, la vitesse maximale $5,20 \mathrm{~m} / \mathrm{s}$, l'accélération maximale $67 \mathrm{~g}$.
IV.2. VAnnes. - Deux vannes spéciales de très grand débit $(200 \mathrm{l} / \mathrm{s})$ et à ouverture et fermeture très rapides $(\sim 12 \mathrm{~ms})$ sont prévues pour la recompression et la détente. Elles seront directement commandées par l'intermédiaire de servovalves suivant un « timing » à définir expérimentalement. Une autre vanne de débit plus faible mais de conception analogue sera utilisée pour la dernière partie de la recompression et le verrouillage. Son ouverture sera asservie à la position $\mathrm{du}$ piston et son fonctionnement devra permettre la recompression correcte des bulles en fin de course et un positionnement constant du piston entre deux détentes.

IV .3. Pompes. - Nous utiliserons des pompes à vis très silencieuses pouvant donner un débit de $601 / \mathrm{mn}$ à 160 bars ( 9 pompes au total). La puissance totale dépensée pour un taux de répétition de $1 \mathrm{~s}$ ne devrait pas dépasser 100 à $120 \mathrm{~kW}$.

IV .4. RÉGULATION DE LA PRESSION DANS LA GHAMBRE. - Un dispositif hydraulique assurera une circulation d'huile dans l'espace intermédiaire et la régulation de la pression dans ce volume. La pression dans la chambre entre deux détentes sera donc indépendante des petites variations éventuelles de température dans le liquide.

V. Conclusion. - Le système de détente présenté ici doit être expérimenté avant la fin 1968. Bien qu'il soit assez classique dans son principe, sa réalisation présente cependant de nombreuses difficultés dues aux dimensions importantes de l'ensemble et à la rapidité du système. Les problèmes d'inertie y jouent un rôle prépondérant, et en particulier l'inertie de l'huile en mouvement doit être réduite au maximum. 